\title{
Effets du Système de Culture sous couverture Végétale (SCV) sur les flux hydriques d'un sol ferrugineux à l'Ouest du Burkina Faso
}

\author{
Baba OUATTARA ${ }^{1}$, Kalifa COULIBALY ${ }^{2,4^{*}}$, Edmond KOHIO ${ }^{3}$, Sory DOUMBIA ${ }^{2}$, \\ Souleymane OUÉDRAOGO${ }^{1}$ et Hassan B. NACRO ${ }^{2}$
}

\author{
${ }^{1}$ Institut de l'Environnement et de Recherches Agricoles (INERA), 01 B.P. 476 Ouagadougou 01, \\ Burkina Faso. \\ ${ }^{2}$ Université Nazi Boni (UNB), Institut du Développement Rural (IDR), Laboratoire d'Etude et de Recherche sur \\ la Fertilité du sol (LERF), 01 B.P. 1091 BoAbo-Dioulasso 01, Burkina Faso. \\ ${ }^{3}$ Centre Universitaire Polytechnique de Dédougou (CUPD), BP 7021 Ouagadougou, Burkina Faso. \\ ${ }^{4}$ Centre International de Recherche Développement sur l'Elevage en zone Subhumide (CIRDES), Unité de \\ Recherche sur les Productions ANimales (URPAN), O1BP 454, Bobo-Dioulasso, Burkina Faso. \\ *Auteur correspondant ; E-mail: kalifacoul1@yahoo.fr
}

\section{REMERCIEMENTS}

Les auteurs remercient la Fondation Internationale pour la Science (FIS) pour l'appui financier.

\section{RÉSUME}

La faible disponibilité de l'eau de pluie et l'érosion, limitent les productions agricoles au Burkina Faso et nécessitent de développer des systèmes de culture innovants. L'étude a comparé les effets du labour conventionnel et du semis direct sous couverture végétale (SCV) sur les flux hydriques d'un sol au Burkina Faso. L'objectif était de déterminer la quantité de paille nécessaire pour limiter l'érosion et améliorer les caractéristiques hydriques du sol. Un dispositif agronomique randomisé comprenant trois répétitions et trois traitements $\left(\mathrm{T}_{0}=\right.$ labour sans paillage, $\mathrm{T}_{2 \mathrm{t}}=$ semis direct sous $2 \mathrm{t} / \mathrm{ha}$ de paillis et $\mathrm{T}_{4 \mathrm{t}}=$ semis direct sous $4 \mathrm{t} / \mathrm{ha}$ de paillis) a été utilisé. Dans ce dispositif, une placette de 1,04 $\mathrm{m}^{2}$ relié à un demi-fût placé sous terre par un tuyau a permis de recueillir et de quantifier les eaux ruisselées et la terre érodée. La perméabilité des traitements a été mesurée par la méthode de l'infiltromètre à double anneaux. Pour mesurer l'humidité du sol, un échantillon par traitement et par horizon a été séché à l'étuve pendant 24 heures à $105{ }^{\circ} \mathrm{C}$. Les résultats montrent que le coefficient de ruissellement a varié de $55,27 \%$ à $16,3 \%$ sous SCV contre $94,1 \%$ sous labour sans paillage. Le SCV a réduit de 76 à $90 \%$ les pertes de terre, a significativement élevé la vitesse d'infiltration comparativement au labour $(\mathrm{P}=0,036)$ et a une meilleure humidité. La quantité de $2 \mathrm{t} / \mathrm{ha}$ de paillis est nécessaire et suffisante pour contrôler le ruissellement et l'érosion, améliorer l'infiltration et conserver une humidité suffisante comparativement au labour.

(C) 2018 International Formulae Group. All rights reserved.

Mots clés: Paillage, semis direct, labour, infiltration, humidité, ruissellement, érosion. 


\title{
Effects of Mulch based Crop Systems (MCS) on water fluxes of a ferruginous soil in the west of Burkina Faso
}

\begin{abstract}
Agricultural productions in Burkina Faso are limited by the weak availability of the rainwater and the erosion. Systems of innovating culture are therefore to develop. The study compared the effects of the conventional ploughing and the direct seedling under plant cover (DSC) on the water fluxes of one soil in Burkina Faso. The objective was to determine the quantity of necessary straw to limit the erosion and to improve the water features of soil. A device agronomic random containing three repetitions and three treatments $\left(\mathrm{T}_{0}=\right.$ ploughing without mulch, $\mathrm{T}_{2 \mathrm{t}}=$ direct seedling under $2 \mathrm{t} / \mathrm{ha}$ mulch and $\mathrm{T}_{4 \mathrm{t}}=$ direct seedling under 4t/ha mulch) has been used. In this device, a plot of $1.04 \mathrm{~m}^{2}$ joined to a half stock placed under ground by a hose, permitted to collect and to quantify water streamed and the eroded soil. The permeability of the treatments has been measured by the method of infiltrometer with duplicate rings. To measure the humidity of soil, a middle sample by treatment and by horizon has been dried at the steam room during 24 hours to $105{ }^{\circ} \mathrm{C}$. The results show that the coefficient of dripping varied from $55.27 \%$ to $16.3 \%$ under DSC against $94.1 \%$ under ploughing without mulch. The DSC reduced 76 to $90 \%$ the losses of soil, raised the speed of infiltration meaningfully compared with the ploughing $(\mathrm{P}=0.036)$ and has a better humidity. The quantity of $2 \mathrm{t} / \mathrm{ha}$ of mulch is necessary and sufficient to control the dripping and the erosion, to improve the infiltration and to keep a sufficient humidity compared with the ploughing.
\end{abstract}

(C) 2018 International Formulae Group. All rights reserved.

Keywords: Mulch, direct seedling, ploughing, infiltration, humidity, dripping, erosion.

\section{INTRODUCTION}

Au Burkina Faso, le secteur agricole est très peu productif si bien qu'il peine à nourrir sa population. Les causes les plus souvent évoquées pour expliquer cette faible performance sont: (i) les conditions climatiques de plus en plus défavorables, (ii) les pratiques agricoles inappropriées (Bado, 2002), (iii) le sous-équipement des agriculteurs et (iv) la pauvreté naturelle même des sols qui les rend susceptibles à l'érosion (Doamba et al., 2011).

La grande variabilité interannuelle et intra saisonnière de la pluie, conduit souvent à des poches de déficit hydrique en saison hivernale, hypothéquant ainsi la production agricole. Or, la majorité de la population du Burkina Faso, est composée de petits producteurs dont les conditions de vie sont basées sur l'agriculture pluviale. Cette irrégulière répartition spatio-temporelle de la pluviosité annuelle moyenne déjà déficitaire, fait de l'agriculture burkinabè une entreprise à risque. Cette situation pourrait davantage s'exacerber au regard de nombreuses simulations agro-climatiques prédisant que le climat éprouvera le secteur agricole subsaharien durant les années à venir (Ouédraogo et al., 2010). On constate aussi qu'une bonne partie de l'eau de pluie qui tombe est perdue par le ruissellement, réduisant ainsi sa disponibilité pour les cultures. Ainsi, plus que la faible pluviosité annuelle, c'est plutôt la faible proportion d'eau de pluie qui atteint l'horizon racinaire des cultures qui est incriminée pour être la principale contrainte à la production ( Sissoko 2009).

En outre, les pratiques agricoles actuelles se caractérisent par la mise en culture continue des terres sans soutien d'une gestion durable de la fertilité des sols. De telles pratiques extensives à travail répété $d u$ sol, à très faibles fertilisants et sans recyclage des résidus de récolte, entraîneraient la dégradation de la fertilité des sols (Bado, 2002) avec notamment la baisse du pH et de la teneure en carbone (Coulibaly et al., 2012).

La faible disponibilité de l'eau à l'échelle de la parcelle et la dégradation continue de la fertilité des sols du fait des pratiques agricoles inappropriées, 
hypothèquent alors la production agricole déjà insuffisante et très instable. La conséquence directe est l'insécurité alimentaire et le mal être des populations (surtout rurales), conduisant parfois à des crises sociopolitiques comme celles dites «émeutes de la faim» qu'ont connues de nombreux pays subsahariens en 2008.

Aujourd'hui, l'un des plus grands défis serait de transformer l'agriculture burkinabè de sorte à accroître durablement sa production, afin de satisfaire les besoins alimentaires et nutritionnels d'une population en perpétuelle croissance, et de dégager des surplus importants pour le marché, pour ainsi procurer des revenus aux populations. Pour relever efficacement ce challenge, dans ce contexte de croissance démographique, de pression foncière et de changements climatiques, il est impératif de développer des modes de production agricole innovants qui contribuent à une gestion conservatoire acceptable du sol et à une mobilisation efficiente de l'eau qui tombe au profit des cultures. Le système de culture sous couverture végétale $(\mathrm{SCV})$, qui tire les principes de «travail minimum et de couverture du sol» du concept de l'agriculture de conservation, se présente alors comme une alternative convenable et prometteuse, en vertu de nombreux avantages qui lui sont connus.

En effet, les travaux conduits sur le système de culture sous couverture végétale (SCV) montrent qu'il permet: (i) d'améliorer l'infiltration et la retention de l'eau du sol et de limiter l'évaporation du sol (Mrabet, 2002, Findeling et al., 2003; Autfray et al.,2007), (ii) de réduire le ruissellement et de protéger le sol contre l'érosion (Autfray et al., 2007; Adekalu et al., 2007; Roose et al., 2008; Dugué, 2014), (iii) d'améliorer la fertilité du sol par l'apport de la matière organique et par la stimulation de l'activité biologique (Scopel et al., 2004; Oorts et al., 2007 ; Sissoko 2009, Dugué, 2014, Ouédraogo et al., 2014). En somme, le SVC est techniquement le système qui pourrait conduire à une intensification écologique de l'agriculture (Djamen et al.,
2015), vivement souhaitée aujourd'hui pour remplacer le système productiviste. Malgré cette importance du SCV, il reste peu pratiqué au Burkina Faso, particulièrement dans sa partie soudanienne, parce qu'insuffisamment connu ou parce que confronté à la compétition pour l'usage des résidus pour le fourrage et pour la couverture du sol. Pour cela, il convient d'évaluer l'effet du niveau de couverture du sol sur les flux hydriques qui s'y déroulent pour dégager les bénéfices correspondants procurés au sol en termes de sa conservation. L'objectif du présent article est de déterminer la quantité de pailles qui suffirait à améliorer la protection du sol contre l'érosion et à mobiliser l'eau à l'échelle de la parcelle au bénéfice des cultures comparativement à la pratique conventionnelle. Ainsi, il sera possible de recommander aux producteurs, la quantité optimale des résidus de récolte à maintenir pour la couverture du sol pour sa gestion conservatoire et le reste pouvant servir à l'alimentation du bétail.

\section{MATERIELS ET METHODES \\ Site d'étude}

L'étude a été conduite à la station expérimentale du Centre International de Recherche Développement sur l'Elevage en zone Subhumide (CIRDES) située à Banakélédaga dans la commune rurale de Bama. Le village de Banakélédaga est situé à $10^{\circ} 11^{\prime} \mathrm{N}, 4^{\circ} 06^{\prime} \mathrm{W}$ et à $300 \mathrm{~m}$ d'altitude. Elle est à une quinzaine de kilomètres de la ville de Bobo-Dioulasso, dans la partie Ouest du Burkina Faso (Figure 1). La commune rurale de Bama dont relève le village de Banakélédaga, a un climat du type sud soudanien (Fontès et Guinko 1995) avec une pluviosité moyenne annuelle oscillant entre 900 à 1200 mm. En 2014 et 2015, la période d'étude, Banakélédaga a enregistré respectivement les hauteurs d'eau de 936,7 $\mathrm{mm}$ et de $962 \mathrm{~mm}$ avec cependant une distribution intra saisonnière très variable des précipitations. Les sols sont de types ferrugineux tropicaux à $\mathrm{pH}$ acide $(5,5$ à 6,5$)$ et à texture limono-sableux à argilo-sableux avec un lessivage actif des éléments nutritifs (BUNASOLS, 1985). Ils ont une bonne 
concentration en bases échangeables et une faible teneur en phosphore (Bado, 1991)

Le système de production agricole est de type extensif pratiqué par des petits paysans faiblement équipés. La production agricole est dominée par la culture des céréales suivie de celle du coton, puis les cultures fruitières notamment la banane et la papaye et les cultures maraîchères. Les rendements sont faibles du fait de la dégradation de la fertilité des sols liée à une pratique de la culture continue des sols suite à une forte pression foncière conduisant à $88 \%$ d'occupation des terres (PCD, 2013).

Quant aux productions animales, qui représentent la seconde activité importante dans la zone, elles sont issues d'un système d'élevage du type extensif, reposant essentiellement sur les rares parcours naturels qui existent encore dans les terroirs. Les résidus culturaux sont ainsi fortement pâturés si bien qu'il en reste très peu pour servir de couverture au sol. Cette faible capacité de charge de l'espace communal oblige les pasteurs à déplacer leurs troupeaux vers le sud dans la région des cascades et vers les républiques de Côte d'Ivoire et du Ghana (PCD, 2013).

\section{Dispositif expérimental}

Un dispositif agronomique à deux facteurs en blocs complètement randomisés comprenant trois répétitions et trois traitements a été utilisé sur un terrain plat à faible pente $(<1 \%)$. Les facteurs considérés étaient le système de culture à labour conventionnel et le semis direct sous couverture végétale ou système de culture sous couverture végétale (SCV) avec deux quantités de pailles. Les parcelles élémentaires ont été de $10 \mathrm{~m}$ x $10 \mathrm{~m}$ séparées entre elles par une allée d'un mètre. Les répétitions ont été des blocs distants de $2 \mathrm{~m}$. Les traitements ont été les suivants:

$>\mathrm{T}_{0}$ : labour conventionnel sans paillage (témoin);

$>\mathrm{T}_{2 \mathrm{t}}$ : semis direct sous $2 \mathrm{t} / \mathrm{ha}$ de paillis;

$>\mathrm{T}_{4 \mathrm{t}}$ : semis direct sous $4 \mathrm{t} / \mathrm{ha}$ de paillis.

\section{Conduite de l'expérimentation}

A l'exception du traitement témoin $\mathrm{T}_{0}$ qui a été labouré à plat et qui n'a pas été couvert par de la biomasse morte, les autres traitements $\left(\mathrm{T}_{2 \mathrm{t}}, \mathrm{T}_{4 \mathrm{t}}\right)$ ont plutôt été seulement couverts par des résidus de culture, notamment les tiges de maïs à différentes quantités (respectivement deux et quatre tonnes par hectare). Un traitement herbicide au round up à la dose 11/ha a été ensuite effectué sur l'ensemble des parcelles avant le semis pour contrôler les adventices. Pour l'étude, la variété de maïs (Zea mays L.) SR21 à cycle intermédiaire (110 jours) et celle précoce de niébé (Vigna unguiculata L.) KVX442 (67 jours) ont été retenues, parce que la SR21 est la variété de maïs communément cultivée dans la zone et la KVX442 parce qu'elle est précoce et a un potentiel de rendement élevé (1,8t/ha). Le maïs a été semé sur toutes les parcelles aux écartements de $40 \mathrm{~cm}$ entre les poquets et de $80 \mathrm{~cm}$ entre les lignes. Le niébé a été semé seulement sur les traitements $\mathrm{SCV}\left(\mathrm{T}_{2 \mathrm{t}}, \mathrm{T}_{4 \mathrm{t}}\right)$ et est intervenu 30 jours après celui du maïs, suivant les mêmes écartements mais entre les lignes du maïs. Sur le maïs, une dose de 150 kg. ha- ${ }^{-1}$ 'engrais composé NPK (15-15-15) a été apportée au $15^{\text {ème }}$ jour après semis (JAS). Puis une dose de $50 \mathrm{~kg}$. ha ${ }^{-1}$ d'urée à $46 \%$ d'azote a été apportée en deux fractions au $30^{\text {ème }}$ JAS et au $45^{\text {ème }}$ JAS. Les doses d'engrais apportées sont celles les plus couramment rencontrées en milieu paysan en culture du maïs dans cette partie du Burkina Faso. Puis, les parcelles ont été sarclées respectivement au $15^{\text {ème }}$ et au $40^{\text {ème }}$ JAS du maïs en 2014 et au 35ème et au $90^{\text {ème } J A S ~ e n ~}$ 2015. La protection phytosanitaire du niébé avec K-Optimal (lambda-cyhalothrine 15g/l et acétamipride $20 \mathrm{~g} / \mathrm{l}$ ) à la dose de $11 . \mathrm{ha}^{-1} \mathrm{a}$ été effectué aux stades de 50\% d'apparition des fleurs et de $50 \%$ de formation des gousses

\section{Mesure du ruissellement et de l'érosion du sol}

Un dispositif de mesure de ruissellement a été placé dans chaque traitement. Il comprenait des feuilles de tôle placées et enfoncées dans le sol tout autour 
d'une placette de $1,04 \mathrm{~m}^{2}$. Ce dispositif a été relié dans le sens de la pente à un demi- fût de 100 litres placé sous terre, par un tuyau. Les mesures ont été effectuées au cours des mois de juillet et d'août 2015, période généralement la plus pluvieuse au Burkina Faso. En effet, huit (08) pluies ayant produit un ruissellement ont été notées à cette date, permettant d'effectuer huit séances de mesure pour chaque traitement. Ainsi, les eaux ruisselées et la terre érodée ont été récupérées dans les fûts après chaque pluie. Ces eaux ont été quantifiées et un échantillon homogénéisé par traitement et par répétition a été prélevé puis décanté et filtré au laboratoire, pour retenir la terre fine entraînée qui a été ensuite séchée puis pesée. Par séance, neuf mesures de quantification d'eau ruisselée ont été effectuées et neuf échantillons d'eau pour la quantification de la terre érodée ont été constitués. La quantité totale de terre érodée a été ensuite calculée par la règle de trois et extrapolée à l'hectare.

\section{Mesure de la perméabilité du sol par l'infiltromètre à double anneaux}

La perméabilité des traitements a été mesurée à l'entrée de la saison hivernale au mois d'avril 2014 et 2015 par la méthode de l'infiltromètre à double anneaux. Il s'agissait d'enfoncer en même temps les deux anneaux dans le sol à une profondeur suffisante pour assurer l'étanchéité entre les deux cellules. L'anneau de garde a ensuite été rempli d'eau jusqu'au niveau requis, pour assurer l'humectation latérale parasite du sol et ainsi permettre d'assimiler le débit de l'anneau interne à une infiltration cylindrique verticale. Les valeurs du front humecté ont été ainsi relevées suivants des intervalles de temps de $10,60,120,180,240$ secondes après que l'eau ait été mise en contact avec le sol des différents traitements. Deux mesures ont été faites sur deux points d'une diagonale de chaque traitement, soit en tout 36 mesures pour l'ensemble. Le coefficient d'infiltration verticale $(\mathrm{Kv})$ a été obtenu par l'expression suivante: $\mathrm{Kv}(\mathrm{m} / \mathrm{s})=\Delta \mathrm{H} / \Delta \mathrm{T}$ où $\Delta \mathrm{H}$ est la profondeur du sol humecté et $\Delta \mathrm{T}$ le temps d'humectation (Savadogo et al., 2007).

\section{Mesure de l'humidité pondérale du sol}

La mesure du taux d'humidité du sol a consisté à effectuer trois prélèvements de sol à l'aide de la tarière manuelle sur les horizons 0 $-10,10-20,20-30,30-40$ et $40-50 \mathrm{~cm}$ suivant une diagonale de chaque parcelle élémentaire (traitement). Durant toutes les semaines du mois de juillet et les deux premières semaines du mois d'octobre, des années 2014 et 2015, un prélèvement a été effectué, puis un échantillon moyen par traitement et par horizon a été retenu et séché à l'étuve (à $105{ }^{\circ} \mathrm{C}$ pendant $24 \mathrm{~h}$ ) jusqu'à obtenir un poids constant. La quantité d'eau a été ensuite déterminée en faisant la différence de poids entre le sol humide et le sol séché. Au total, douze séances de prélèvement ont eu lieu et il a été ainsi traité 45 échantillons à chaque séance, qui ont permis d'obtenir les taux d'humidités moyens par traitement .Une moyenne des valeurs obtenues par traitement, puis par répétition a été déterminée pour réduire les marges d'erreurs. Ces moyennes ont servi par la suite à représenter et à comparer le taux d'humidité des traitements. Les prélèvements ont été effectués les 9,16, 23 et 30 juillet et les 8 et 15 octobre en 2014 et en 2015, ils ont été réalisés les 7, 14, 21 et 28 juillet et les 7 et 14 octobre. Etant entendu que les mois d'août et de septembre sont les plus pluvieux, il n'y a pas eu de prélèvement durant cette période, à cause de l'excès d'humidité qui n'aurait pas permis d'observer un effet significatif sur les différents traitements du point de vue humidité du sol. Le taux d'humidité a été obtenu à partir de la formule suivante :

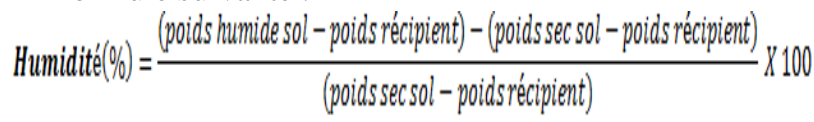

\section{Analyse des données}

Les données collectées ont été saisies à l'aide du logiciel EXCEL version 2010 qui a servi aussi pour générer les graphiques. Le logiciel XLSTAT version 7.5.2 a été utilisé pour l'analyse statistique des données. Le test de Fisher a permis la comparaison des moyennes au seuil de 5\%. 


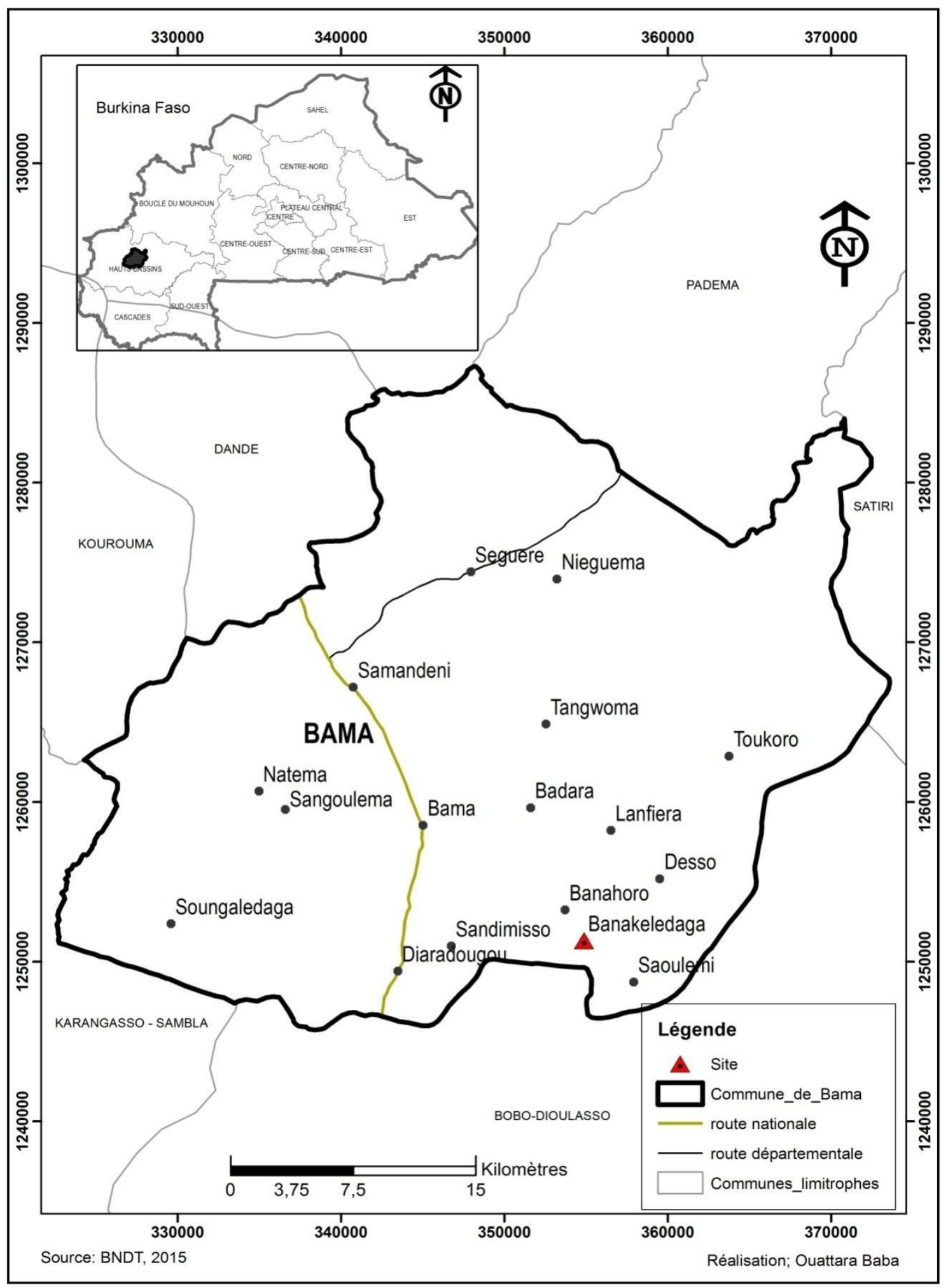

Figure 1: Localisation de Banakélédaga. 


\section{RÉSULTATS}

Effet du Semis direct sous couverture végétale sur le ruissellement et l'érosion du sol

Le ruissellement et l'érosion ont été réduits par le semis direct sous couverture $T_{2 t}$ et $\mathrm{T}_{4 \mathrm{t}}$ comparativement au labour conventionnel $\left(\mathrm{T}_{0}\right)$. Cette réduction a été respectivement de 75,65 à 87,67\% pour $\mathrm{T}_{2 \mathrm{t}}$ et $\mathrm{T}_{4 \mathrm{t}}$ (Tableau 1). Le ruissellement cumulé (CumRuiss) et l'érosion du sol ont significativement baissé $(\mathrm{P}=0,0001)$ avec l'apport de $4 \mathrm{t}$ MS/ha de paille $(67,31 \mathrm{~mm}$ d'eau et $653,44 \mathrm{~kg}$ de terre) par rapport au témoin $\mathrm{T}_{0}$ sans paillage $(388,65 \mathrm{~mm}$ d'eau et $6327,71 \mathrm{~kg}$ de terre). Ainsi, le taux moyen de réduction du ruissellement et de l'érosion par les traitements SCV ont été respectivement de $41,27 \%\left(\mathrm{~T}_{2 \mathrm{t}}\right)$ à $82,68 \%\left(\mathrm{~T}_{4 \mathrm{t}}\right)$ et de $75,65 \%\left(\mathrm{~T}_{2 \mathrm{t}}\right)$ à $89,67 \%\left(\mathrm{~T}_{4 \mathrm{t}}\right)$ par rapport au témoin à labour sans paillage $\left(\mathrm{T}_{0}\right)$. On observe que la réduction du ruissellement et de l'érosion a été d'autant importante que la quantité de paille utilisée en couverture du sol était plus élevée. Le paillage avec $4 \mathrm{t} / \mathrm{ha}$ de tiges de maïs $\left(\mathrm{T}_{4 \mathrm{t}}\right)$ a ainsi réduit le ruissellent de $70,51 \%$ et l'érosion de 57,58\% par rapport à celui de $2 \mathrm{t} / \mathrm{ha}\left(\mathrm{T}_{2 \mathrm{t}}\right)$.

\section{Effet du semis direct sous couverture végétale (SCV) sur l'infiltration verticale de l'eau dans le sol}

La vitesse de l'infiltration verticale de l'eau dans le sol a été plus élevée en première année d'expérimentation $(0,08$ à $0,12 \mathrm{~cm} / \mathrm{s})$ qu'en deuxième année $(0,02$ à $0,04 \mathrm{~cm} / \mathrm{s})$. Elle n'a été significativement différente $(\mathrm{P}=$ 0,036 ) entre les traitements qu'en seconde année de l'expérimentation en 2015 (Tableau 2). Cette vitesse a été nettement plus élevée sur les parcelles en SCV que celles en labour conventionnel $\left(\mathrm{T}_{0}\right)$. La meilleure vitesse d'infiltration de l'eau dans le sol $(0,12 \pm 0,21$ $\mathrm{cm} / \mathrm{s}$ ) a été obtenue avec le niveau de paillage de $2 \mathrm{t} / \mathrm{ha}\left(\mathrm{T}_{2 \mathrm{t}}\right)$ en 2014.

\section{Effet du semis direct sous couverture végétale sur l'humidité pondérale du sol}

La Figure 2 présente les profils hydriques du sol selon le travail du sol et les quantités de résidus utilisés en paillage du sol au cours des deux années d'expérimentation (2014, 2015). De façon générale, ces résultats indiquent, indépendamment de la profondeur $\mathrm{du}$ sol, une tendance des traitements SCV $\left(\mathrm{T}_{2 \mathrm{t}}\right.$ et $\mathrm{T}_{4 t}$ ) à engendrer une humidité plus élevée que celle du traitement témoin $\left(\mathrm{T}_{0}\right)$ en labour conventionnel. Le taux d'humidité moyen du niveau de paillage $4 \mathrm{t} / \mathrm{ha}\left(\mathrm{T}_{4 \mathrm{t}}\right)$, s'est révélé supérieur à celui du niveau de paillage $2 \mathrm{t} / \mathrm{ha}$ $\left(T_{2 t}\right)$. Pour les prélèvements effectués les 23 et 30 juillet 2014 et le 8 octobre 2014 (Figure 2C14, D14 et E14), les sols des traitements $\mathrm{SCV}\left(\mathrm{T}_{2 \mathrm{t}}\right.$ et $\left.\mathrm{T}_{4 \mathrm{t}}\right)$ ont été significativement $(\mathrm{P}<$ $0,05)$ plus humides que ceux du témoin à labour conventionnelle $\left(\mathrm{T}_{0}\right)$.La différence entre les traitements $T_{2 t}$ et $T_{4 t} n$ 'a pas été significative $(\mathrm{P}>0,05)$.

\section{DISCUSSION}

Le ruissellement et l'érosion observés sont statistiquement plus faibles sur les traitements SCV comparativement au témoin en labour conventionnel, ce qui confirme les travaux de Douzet (2010). En effet, le paillage, en formant un écran entre le sol et les gouttes d'eau de pluie, a non seulement réduit l'arrachage des particules de terre, mais a également constitué une barrière physique à l'écoulement de l'eau sur le sol. Il a alors ralenti la vitesse du ruissellement, diminuant ainsi le déplacement des particules de sol arrachées. Des résultats similaires ont été trouvés par Scopel et al. (2005), et Sissoko (2009), qui expliquaient que la protection permanente du sol avec une couverture vivante ou morte était une méthode efficace pour contrôler le ruissellement et l'érosion des sols. Aussi, des études plus récentes (Dugué et al., 2015), ont également montré la performance des SCV à réduire le ruissellement et l'érosion hydrique.

Les valeurs du coefficient de ruissellement annuel moyen obtenues $(94,10 \%, 55,27 \%$ et à $16,30 \%$, respectivement pour le traitement conventionnel, le traitement avec $2 \mathrm{t} / \mathrm{ha}$ de paille et le traitement avec $4 \mathrm{t} / \mathrm{ha}$ de paille) sont nettement supérieures à celles de Scopel et al. (2005) qui ont obtenu $49 \%$ pour le témoin contre $7 \%$ pour un SCV avec $4,5 \mathrm{t} / \mathrm{ha}$ de paillage. La forte sensibilité du sol du site 
Tableau 1: Effet du SCV sur le ruissellement et l'érosion du sol.

\begin{tabular}{lllllll}
\hline Facteurs & \multicolumn{1}{c}{$\mathbf{T}_{\mathbf{0}}$} & \multicolumn{1}{c}{$\mathbf{T}_{\mathbf{2 t}}$} & $\mathbf{T}_{\mathbf{4 t}}$ & ddl & P & S \\
& & & & & & \\
\hline CumRuiss (mm) & $388,65 \pm 12,28$ & $228,27 \mathrm{~b} \pm 11,62$ & $67,31 \mathrm{c} \pm 6,68$ & 2 & 0,0001 & THS \\
RéducRuiss (\%) & 0,00 & 41,27 & 82,68 & & & \\
Cram(\%) & 94,10 & 55,27 & 16,30 & & & \\
Erosion du sol & $6327,71 \mathrm{a} \pm 269$ & $1540,51 \mathrm{~b} \pm 104,03$ & $653,44 \mathrm{c} \pm 78,34$ & 2 & 0,0001 & THS \\
(kg/ha) & & & & & & \\
& & & & & & \\
RéducEros (\%) & 0,00 & 75,65 & 89,67 & & & \\
\end{tabular}

CumRuiss :accrued dripping ; ruissellementcumulé; RéducRuiss : reduction of dripping ; réduction du ruissellement; Cram: yearly drippingcoefficient average ; coéfficient du ruissellementannuelmoyen;RéducEros:reduction of erosion; réduction de l'érosion; THS : very highly meaningful; trèshautementsignificatif

Tableau 2: vitesse d'infiltration verticale de l'eau dans le sol en fonction des traitements en 2014 et 2015.

\begin{tabular}{|c|c|c|}
\hline \multirow{2}{*}{ Traitements } & \multicolumn{2}{|c|}{ Vitesse $(\mathrm{cm} / \mathrm{s})$} \\
\hline & 2014 & 2015 \\
\hline $\mathrm{T}_{0}$ & $0,11 \pm 0,20$ & $0,02 b \pm 0,02$ \\
\hline $\mathrm{T}_{2 \mathrm{t}}$ & $0,12 \pm 0,21$ & $0,04 a \pm 0,04$ \\
\hline $\mathrm{T}_{4 \mathrm{t}}$ & $0,08 \pm 0,13$ & $0,03 \mathrm{ab} \pm 0,01$ \\
\hline F de Fisher & 0,446 & 3,405 \\
\hline $\operatorname{Pr}>\mathrm{F}$ & 0,642 & 0,036 \\
\hline Signification & NS & $\mathrm{S}$ \\
\hline
\end{tabular}


B. OUATTARA et al. / Int. J. Biol. Chem. Sci. 12(4): 1770-1783, 2018




B. OUATTARA et al. / Int. J. Biol. Chem. Sci. 12(4): 1770-1783, 2018

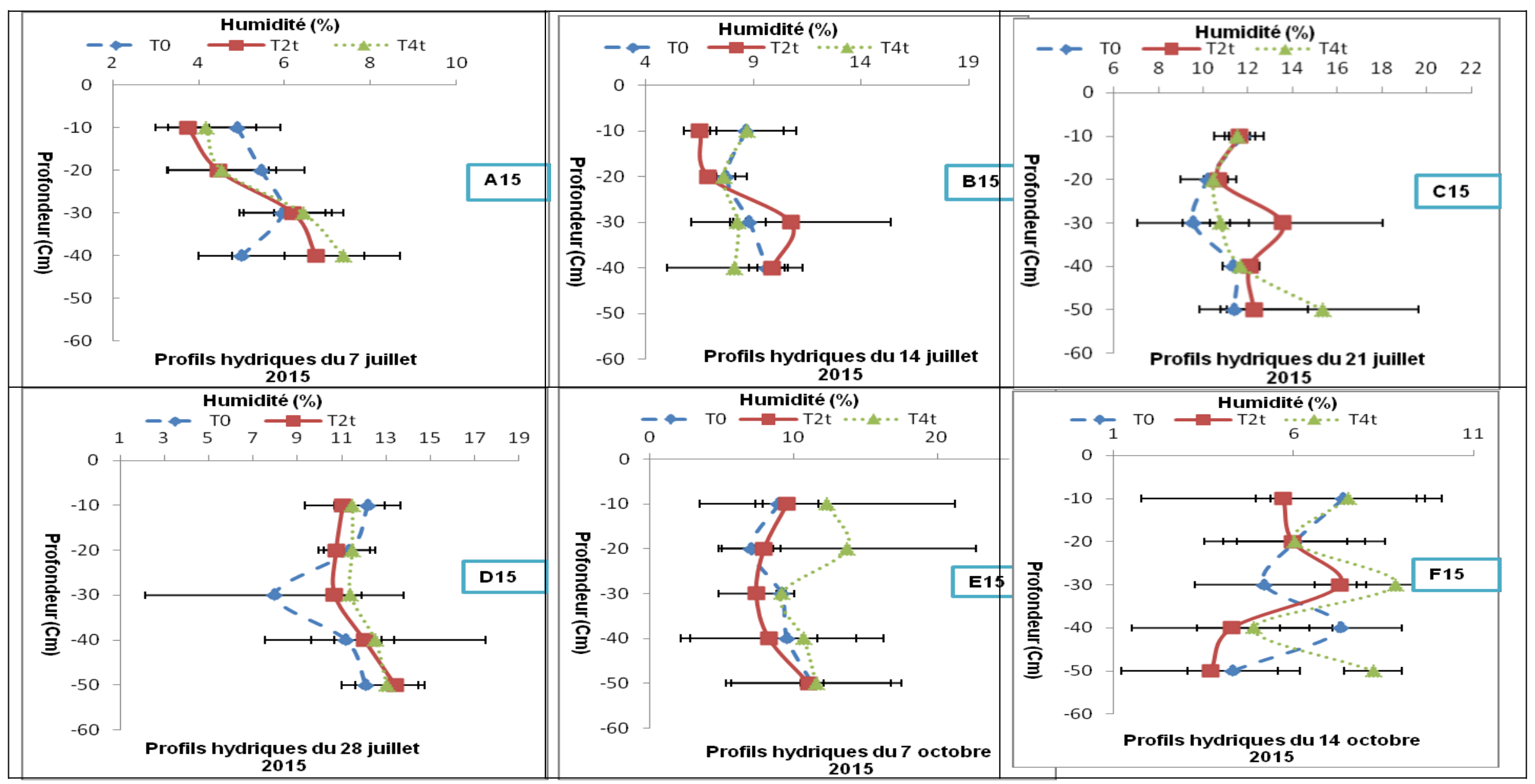

Figure 2: Profils hydriques du sol des mois de juillet et d'octobre des années 2014 et 2015. 
de l'étude au ruissellement, s'expliquerait par sa pauvreté en argile et en matière organique. Nos résultats confirment la tendance à la réduction du coefficient de ruissellement par le SCV par rapport au labour conventionnel, constatée par Scopel (2005).

$$
\text { L'observation d'une vitesse }
$$

d'infiltration de l'eau plus élevée avec les traitements SCV, pourrait s'expliquer par le développement de l'activité biologique occasionnée par la présence des résidus sur le sol. En effet, Sissoko (2009), expliquait que le paillage, par la température agréable qu'elle conservait, constituerait un refuge pour la méso-pédo-faune qui fore des galeries dans le sol, facilitant ainsi l'infiltration de l'eau de pluie dans le profil du sol. Aussi, la présence des résidus, en ralentissant le ruissellement, augmente le temps de séjour de l'eau sur le sol et favoriserait donc l'infiltration. Moussadek (2011) indiquait ainsi, que l'infiltration augmentait de $54 \%$ lorsque $45 \%$ des résidus étaient laissés au sol.

Par ailleurs, avec les traitements SCV humide dans le sol a été plus importante qu'avec le témoin $\left(\mathrm{T}_{0}\right)$ en labour sans aucune couverture du sol. Le paillage a donc permis d'accroître le volume d'eau par la réduction de l'évaporation de l'eau infiltrée dans le sol.

De nombreux auteurs sont parvenus à des résultats similaires indiquant une meilleure conservation de l'humidité du sol par le SCV par rapport au labour conventionnel. En effet, Dounias (2001) indiquait que l'évaporation du sol en semis direct avec paillis était faible $(<50 \mathrm{~mm})$ quelles que soient les conditions climatiques et restait bien inférieure à celle d'un labour sans couverture. Cet effet de la couverture sur le gain d'humidité du sol a été aussi confirmé par Scopel et al. (2005) qui rapportent que le paillage a un effet positif sur l'évaporation directe de l'ensemble sol- résidus et qu'il réduirait l'évaporation de 10 à $20 \%$ environ. Une étude de Djamen et al. (2015) au Burkina Faso, a également montré qu'une couverture de 4 tonnes de résidus par hectare limiterait l'évaporation de l'eau du sol. Celle de Dugué et al. (2015) a indiqué les performances avérées des SCV dans un meilleur stockage de l'eau dans le sol. Elle soutenue par Sermé et al. (2015) qui ont concluent que l'application du paillage augmentait le stock en eau du sol.

Cependant, l'examen de la pluviométrie de l'année 2014 montre que l'humidité à la date du 23 juillet (Figure 2C14), du 30 juillet (Figure 2-D14) et du 08 octobre (Figure 2-C14), déterminées respectivement après les pluies du 18 juillet (18 mm), 25 juillet ( $34 \mathrm{~mm})$ et 03 octobre (25 $\mathrm{mm}$ ) ayant induit une différence significative entre l'humidité du SCV et celle du témoin labouré ont été mesurées 5 jours après une pluie. Cela suggère que l'amélioration de l'humidité par le SCV n'est perceptible qu'après une poche de sécheresse d'au moins cinq jours. Cette analyse révèle aussi que la différence d'humidité est d'autant plus prononcée entre le SCV et le labour conventionnel, que la hauteur d'eau tombée est faible.

\section{Conclusion}

L'étude a montré la performance du SCV à augmenter le stock en eau du sol, par l'amélioration de l'infiltration de l'eau dans le sol et par la réduction du ruissellement et de l'érosion qu'il induit. Les résultats indiquent, que par rapport au labour, les SCV ont un effet bénéfique sur l'humidité du sol qui est d'autant marquée que la période de sécheresse est plus prononcée. Le facteur de l'amélioration de la vitesse d'infiltration varie de 1,5 à 2 alors que la réduction du ruissellement et de l'érosion par le SCV sont ainsi respectivement de 41 à $83 \%$ et de 76 à $90 \%$ par rapport au système conventionnel. 
Cette performance est à attribuer au paillage qui protège le sol contre les effets (splah, évaporation, ruissellement) des intempéries, favorise une intensification de l'activité biologique améliorant la porosité du sol au bénéfice de l'infiltration, et rend difficile le détachement et le transport des particules de sol en se posant en obstacle aux gouttes d'eau de pluie et à leur écoulement.

En zone sud soudanienne du Burkina Faso, où les poches de sécheresse sont récurrentes et où les eaux tombées profitent peu aux cultures, parce que le plus souvent perdues par ruissellement, cette performance du SCV dans la gestion des flux hydriques, parait être un avantage certain. En effet, l'amélioration des caractéristiques physiques du sol pourrait augmenter sa capacité productive et présager ainsi une meilleure production. Ces travaux suscitent alors la nécessité de poursuivre l'étude pour mesurer l'effet de cet avantage sur les rendements agronomiques et la fertilité chimique et biologique du sol.

\section{CONFLIT D'INTÉRÊTS}

Tous les auteurs ont pris connaissance de la dernière version du manuscrit et ont donné leur accord pour soumission. Les auteurs déclarent qu'il n'y a aucun conflit d'intérêt.

\section{CONTRIBUTION DES AUTEURS}

BO a conçu le protocole et le dispositif expérimental, a participé à sa mise en place et a traité les données et a rédigé le projet d'article; $\mathrm{KC}$ a participé à la conception du protocole et du dispositif expérimental, à sa mise en place et à la rédaction de l'article. $\mathrm{EK}$ et $\mathrm{SD}$ ont aidé au suivi de l'expérimentation et à la collecte des données ; HBN a aidé à la correction du protocole et du projet d'article ; SO a facilité les travaux en laboratoire.

\section{RÉFÉRENCES}

Adekalu KO, Olorunfemi LA, Osunbitan JA. 2007. Grass mulching effect on infiltration surface runoff and soil loss of three agricultural soils in Nigeria . Bioresource Technology, 98: 912917.

DOI:

https://doi.org/10.1016/j.biortech.2006.0 2.044

Autfray P, Sissoko F, Keita A, Konaté CM. 2007. SCV et agronomie dans les systèmes cotonniers. Présentation du projet SCV Mali. In : Agro écologie et Techniques Innovantes dans les Systèmes de Production Cotonniers. Actes du séminaire, septembre 2007, SODECOTON, Projet ESA, Maroua, Cameroun, p.16.

Bado BV. 2002. Rôle des légumineuses sur la fertilité des sols ferrugineux tropicaux des zones guinéenne et soudanienne du Burkina Faso. Thèse de doctorat : Université Laval-Québec, p.197

BUNASOLS. 1985. Etat de connaissance de la fertilité des sols au Burkina Faso. Document technique No1, p.50

Coulibaly K, Vall E, Autfray P, Nacro HB, Sedogo PM. 2012. Effets de la culture permanente coton-maïs sur l'évolution d'indicateurs de fertilité des sols de l'Ouest du Burkina Faso. Int. J. Biol. Chem. Sci., 6(3): 1069-1080. DOI: http://dx.doi.org/10.4314/ijbcs.v6i3.13

Djamen NP, Andrieu N, Zerbo I, Ouédraogo Y, Le Gal PY. 2015. Agriculture de conservation et performances 
des exploitations agricoles en Afrique de l'Ouest. CahAgric., 24: 113-122.

DOI:

https://doi.org/10.1684/agr.2015.0743

Doamba SMF, Nacro HB, Sanon A, Sedogo

PM. 2011. Effet des cordons pierreux sur l'activité biologique d'un sol ferrugineux tropical lessivé (Province du Kouritenga au Burkina Faso). Int. J. Biol. Chem. Sci., 5(1): 304-313 DOI: http://dx.doi.org/10.4314/ijbcs.v5i1.6806 5

Douzet JM, Scopel E, Muller B, Rakotoarisoa J, Albrecht A, Drazafindramanana NC. 2010. Effets des systèmes de cultures en semis direct avec couverture végétale sur le ruissellement et l'érosion des cultures pluviales des Hautes Terres de Madagascar. Etude et Gestion des Sols Association française pour l'étude des sols, 2010, 17 (2), p.131-142. 〈cirad$00762983\rangle$

Dugué P. 2014. Semis direct, Système de culture sous couverture végétale et agroécologie, vers où va-t-on? Quelques enseignements de l'atelier de capitalisation sur les impacts et effets des systèmes de culture sous couvert végétal. Programme d'appui multi-pays à l'agroécologie. Montpellier, Agropolis, du 6 au 8 novembre 2013. Alternatives Rurales (1) ; p.5

Dugué P, Djamen NP, Faure G, Le Gal PY. 2015. Dynamiques d'adoption de l'agriculture conservation dans les exploitations familiales : de la technique aux processus d'innovation. CahAgric., 24: 60-68.

DOI: http://dx.doi.org/10.1684/agr.2015.0748

Dounias I. 2001. Etudes et Travaux ${ }^{\circ}{ }^{19}$, les systèmes de culture à base de couverture végétale et semis direct en zones tropicales. Synthèse bibliographique, Centre National d'Etudes Agronomiques des Régions Chaudes, p.164

Findeling A, Ruy S, Scopel E. 2003. Modeling the effects of a partial residue mulch on runoff using a physically based approach. J. Hydrol., 275: 49-66. DOI: 10.1016/S0022-1694(03)00021-0

Fontès J, Guinko S. 1995. Carte de la végétation et de l'occupation du sol du Burkina Faso. Notice explicative. Ministère de la coopération Française. Projet Campus (88 313 101) p.57

Lal R. 1975. Role of mulching techniques in tropical soil and water management. Technical Bulletin IITA, Ibadan.

Mrabet R. 2002. Stratification of soil aggregation and organic matter under conservation tillage systems in Africa. Soil Tillage Res., 66: 119-128. DOI: 10.1016/S0167-1987(02)00020-X

Moussadek R, Mrabet R, Zante P, Lamachère JM, Pépin Y, Le

Bissonnais Y, Ye L, Verdoodt A, Van Ranst E. 2011. Effets du travail du sol et de la gestion des résidus sur les propriétés du sol et sur l'érosion hydrique d'un Vertisol Méditerranéen. Can. J. Soil Sci., 91: 627-635. DOI: 10.4141/cjss 10096

Ouédraogo J, Ouédraogo E, Nacro HB. 2014. Effet de l'interaction entre des modes de gestion de fertilité et la macrofaune sur la productivité du niébé et du sorgho en zone nord soudanienne du Burkina Faso. Int. J. Biol. Chem. Sci., 8(1): 104-114. DOI: http://dx.doi.org/10.4314/ijbcs.v8i1.10 PCD, 2013.

Roose E. 1981. Dynamique actuelle de sols ferralitiques et ferrugineux tropicaux d'Afrique Occidentale. Étude expérimentale des transferts hydrologiques et biologiques de matières sous végétations naturelles ou cultivées. Collection Travaux et Documents 130. Paris : Orstom éditions, $1981 ;$ p.567

Roose E, Blavet D, Sabir M, Ouagga T, Cheggour A, Simonneaux V, Olivier R, Ferrer H, Louri J, Chotte JL. 2008. Influence de l'utilisation d'un sol brun vertique sur les stocks de carbone $\mathrm{du}$ sol, les risques de ruissellement et d'érosion et le devenir du carbone érodé 
au Maroc. P.317-324 dans E. Roose and J. Albergel, éd. Efficacité de la GCES en milieu semi arides. AUF, EAC et IRD éditeurs Paris, France.

Savadogo P, Sawadogo L, Tiveau T. 2007. Effects of grazing intensity and prescribed fire on soil physical and hydrological properties and pasture yield in the savanna woodlands of Burkina Faso. Agriculture, Ecosystems and Environment, 118: 80-92. DOI: $10.1016 /$ j.agee.2006.05.002

Sermé I, Ouattara K, Logah V, Taonda JB, Palé S, Quansah C, Abaidoo CR. 2015. Impact of tillage and fertility management options on selected soil physical properties and sorghum yield. Int. J. Biol. Chem. Sci., 9(3): 1154-1170. DOI: http://dx.doi.org/10.4314/ijbcs.v9i3.2

Scopel E, Macena F, Corbeels M, Affholder F, Maraux F. 2004. Modeling crop residue mulching effects on water use and production of maize under semi-arid and humid tropical conditions.

Agronomy, 24: 383-395. DOI: 10.1051/agro: 2004029
Scopel E, Douzet JM, Silva FAM, Cardoso A, Moreira JAA, Findeling A, Bemoux M. 2005. Impact des systèmes de culture en semis direct avec couverture végétale (SCV) sur la dynamique de l'eau, de l'azote minéral et du carbone du sol dans les cerrados brésiliens. Cah Agric., 14 (1): 71-75.

Sissoko F. 2009. Analyse des flux d'eau dans les systèmes de culture sous couverture végétale en zone soudano sahélienne: cas du coton semé après une culture de sorgho/Brachiaria au sud du Mali. Thèse de doctorat de Sup Agro, Montpellier, p.169

Oorts K, Bossuyt H, Labreuche J, Merckx R, Nicolardot B. 2007. Carbon and nitrogen stocks in relation to organic matter fractions, aggregation and pore size distribution in no-tillage and conventional tillage in northern France.Eu $J$ Soil Sci., 58 : 248259. DOI:

10.1111/j.1365-2389.2006.00832.x

Ouédraogo M, Dembélé Y, Somé L. 2010. Perceptions et stratégies d'adaptation aux changements des précipitations : cas des paysans du Burkina Faso. Science et Changements planétaires/Sécheresse, 21(2): 87-96. DOI: $10.1684 / \mathrm{sec} .2010 .0244$ 